\title{
Bifurcation for the constant scalar curvature equation and harmonic Riemannian submersions
}

\author{
Nobuhiko Otoba* Jimmy Petean ${ }^{\dagger}$
}

\begin{abstract}
We study bifurcation for the constant scalar curvature equation along a one-parameter family of Riemannian metrics on the total space of a harmonic Riemannian submersion. We provide an existence theorem for bifurcation points and a criterion to see that the conformal factors corresponding to the bifurcated metrics must be indeed constant along the fibers. In the case of the canonical variation of a Riemannian submersion with totally geodesic fibers, we characterize discreteness of the set of all degeneracy points along the family and give a sufficient condition to guarantee that bifurcation necessarily occurs at every point where the linearized equation has a nontrivial solution. In the model case of quaternionic Hopf fibrations, we show that symmetry-breaking bifurcation does not occur except at the round metric.
\end{abstract}

\section{Introduction}

It is well known that every conformal class on a closed manifold carries a Riemannian metric of constant scalar curvature (cf. Yamabe [23], Trudinger [21], Aubin [1], Schoen [18]) while such metrics of unit volume within a conformal class are not necessarily unique (e.g. Kobayashi [11], Schoen [19]). More recently, de Lima-Piccione-Zedda [7] introduced a setup of bifurcation problem for the constant scalar curvature equation and studied direct product Riemannian manifolds from this perspective. Related work about local bifurcation on the total space of a Riemannian submersion with totally geodesic fibers include [4], [5]. For global aspects of bifurcation in this context, see [13], [16], [8].

In this article, we study harmonic Riemannian submersions of constant scalar curvature and show in particular the following. Let $(F, t \hat{g}) \rightarrow\left(M^{m}, g(t)\right) \stackrel{\varphi}{\rightarrow}$

\footnotetext{
*Universität Regensburg, 93040 Regensburg, Deutschland. nobuhiko.otoba@ur.de

${ }^{\dagger}$ Centro de Investigación en Matemáticas, Jalisco S/N, Col. Valenciana CP: 36023 Guanajuato, Gto, México. jimmy@cimat.mx
} 
$(N, h)$ be the canonical variation of a Riemannian submersion with totally geodesic fibers, $m \geq 3$. Assume both the typical fiber $(F, \hat{g})$ and the base space $(N, h)$ have constant scalar curvature, so that $g(t)$ has constant scalar curvature for each $t \in(0, \infty)$. We denote by $\boldsymbol{s}_{\hat{g}}$ and $\lambda_{1}\left(-\Delta_{\hat{g}}\right)$ the scalar curvature and the first nonzero eigenvalue of the positive Laplacian acting on functions for the metric $\hat{g}$, respectively. Also, let $B \subset(0, \infty)$ be the set of all bifurcation instants for the constant scalar curvature equation along the family $\{g(t)\}_{t>0}$.

Theorem 1.1. If $\boldsymbol{s}_{\hat{g}}>0$, then there exists a sequence $\left\{b_{l}\right\}_{l \geq 1} \subset B$ such that $b_{l+1}<b_{l}$ for all $j$ and $\lim _{l \rightarrow \infty} b_{l}=0$. If moreover $\lambda_{1}\left(-\Delta_{\hat{g}}\right)>\boldsymbol{s}_{\hat{g}} /(m-1)$, then there exists a real number $\varepsilon>0$ so that the following hold:

(1) $B \cap(0, \varepsilon)=\left\{b_{j} \mid j\right.$ large $\}$. That is, bifurcation only occurs at $b_{j}$ 's for $t$ sufficiently small.

(2) If $b \in B \cap(0, \varepsilon)$ and if $\tilde{g}=e^{2 f} g(b)$ is a constant scalar curvature metric sufficiently close to $g(b)$, then the conformal factor $f$ has to be constant along the fibers of $\varphi$.

Also, $B$ is discrete if $\boldsymbol{s}_{\hat{g}} /(m-1)$ is not a nonzero eigenvalue of $-\Delta_{\hat{g}}$.

This article is organized as follows. We define the bifurcation points for the constant scalar curvature equation along a general one-parameter family of Riemannian metrics in Sect. 2. In Sect. 3, we introduce a double Lyapunov-Schmidt reduction (Lemma 3.1) and prove a theorem whose conclusion is that the conformal factors have to be constant along the fibers of the submersion in concern (Theorem 3.2). We show existence of bifurcation points in Sect. 4. The results in Sects. 3, 4 may be applied to harmonic Riemannian submersions. In Sect. 5, we specialize to the case of Riemannian submersions with totally geodesic fibers and show Theorem 5.5, which is a slightly refined version of Theorem 1.1.

\section{A setup for bifurcation of constant scalar curvature metrics}

Let $\{g(t)\}_{t \in I}$ be a $C^{\infty}$ family of constant scalar curvature metrics on a closed manifold $M^{m}, m \geq 3$. Here, $I$ is an open interval of $\mathbb{R}$, and the scalar curvature $\boldsymbol{s}_{g(t)}$ may depend on $t$. For each $t$, consider the PDE

$$
-a_{m} \Delta_{g(t)} u+s_{g(t)}\left(u-u^{p_{m}-1}\right)=0
$$

for $u \in C_{+}^{k+2, \alpha}(M, g(t))=\left\{u \in C^{k+2, \alpha}(M, g(t)) \mid u>0\right\}$, which is the EulerLagrange equation of the functional $E_{g(t)}: C_{+}^{k+2, \alpha}(M, g(t)) \rightarrow \mathbb{R}$ defined by

$$
E_{g(t)}(u)=\int_{M} \frac{a_{m}}{2}|d u|^{2}+\boldsymbol{s}_{g(t)}\left(\frac{u^{2}}{2}-\frac{u^{p_{m}}}{p_{m}}\right) d \mu_{g(t)} .
$$


Here, $a_{m}=\frac{4(m-1)}{m-2}, p_{m}=\frac{2 m}{m-2}$. We are concerned with the bifurcation phenomena for the family $C_{+}^{k+2, \alpha}(M) \times I \rightarrow C^{k, \alpha}(M)$ of potential operators defined by the left hand side of (2.1). Since the Banach spaces $C^{l, \alpha}\left(M, g\left(t_{1}\right)\right), C^{l, \alpha}\left(M, g\left(t_{2}\right)\right)$ and the Hilbert spaces $L^{2}\left(M, g\left(t_{1}\right)\right), L^{2}\left(M, g\left(t_{2}\right)\right)$ for $t_{1}, t_{2} \in I$ are isomorphic but not necessarily uniformly for all $t \in I$, respectively, it seems to be crucial for the study of global bifurcation along the family $\{g(t)\}$ to take into account, as in de Lima-Piccione-Zedda [7, Appendix A], the change of function spaces as $t$ varies. However, as long as bifurcation local in $t$ is concerned, the following setup is convenient.

Definition 2.1. For $t_{\star} \in I$, we say $g\left(t_{\star}\right)$ is a bifurcation point for the constant scalar curvature equation along $\{g(t)\}_{t \in I}$ if there exist sequences $\left\{t_{j}\right\}_{j \geq 1} \subset I$, $\left\{u_{j}\right\}_{j \geq 1} \subset C^{k+2, \alpha}\left(M, g\left(t_{\star}\right)\right)$ such that $u_{j}$ is a nonconstant solution to (2.1) for each $j$ and $t_{j} \rightarrow t_{\star}, u_{j} \rightarrow 1$ as $j \rightarrow \infty$.

Recall that the scalar curvature of the conformally deformed metric $u^{p_{m}-2} g$, $u \in C_{+}^{2}(M)$ is equal to $u^{1-p_{m}}\left(-a_{m} \Delta_{g} u+\boldsymbol{s}_{g} u\right)$. Since the elliptic regularity for $W^{1,2_{-}}$ critical equations due to Trudinger [21] shows that every solution of $C_{+}^{k+2, \alpha}(M, g)$ to $-a_{m} \Delta_{g} u+\boldsymbol{s}_{g}\left(u-u^{p_{m}-1}\right)=0$ is indeed $C^{\infty}$ smooth, $g\left(t_{\star}\right)$ is a bifurcation point according to Definition 2.1 if and only if $t_{\star}$ is a bifurcation instant in the sense of de Lima-Piccione-Zedda [7, pp. 264-265], provided that sufficiently high regularity is assumed. Note that their volume normalization of the constant scalar curvature metrics is equivalent to our requirement that the conformal factors $u_{j}$ be nonconstant.

\section{A double Lyapunov-Schmidt reduction}

Let $X^{2}, Y^{2}$ Banach spaces and $\iota_{X}: X^{1} \rightarrow X^{2}, \iota_{Y}: Y^{1} \rightarrow Y^{2}$ the inclusions of closed linear subspaces $X^{1} \subset X^{2}, Y^{1} \subset Y^{2}$. Also, let $U^{2}$ be an open neighborhood in $X^{2}$ of some $x_{0} \in X^{1}, U^{1}=U^{2} \cap X^{1}$, and $S^{i}: U^{i} \rightarrow Y^{i}$ a $C^{1}$ map such that $S^{i}\left(x_{0}\right)=0$ and that the diagram

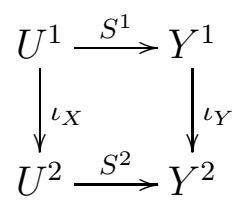

commutes. Assume $L^{i}:=\left.d S^{i}\right|_{x_{0}}: X^{i} \rightarrow Y^{i}$ is Fredholm, so that one can perform the Lyapunov-Schmidt reduction for the equation $S^{i}\left(x^{i}\right)=0$ near $x_{0}$ for each $i=$ 1,2. Note that ker $L^{1} \subset \operatorname{ker} L^{2}$ and $\operatorname{ran} L^{1} \subset \operatorname{ran} L^{2} \cap Y^{1}$ hold since commutativity of (3.1) implies $\iota_{Y} \circ L^{1}=L^{2} \circ \iota_{X}$. 
Lemma 3.1. Suppose $\operatorname{ker} L^{1}=\operatorname{ker} L^{2}, \operatorname{ran} L^{1}=\operatorname{ran} L^{2} \cap Y^{1}$, and a linear subspace $W$ of $Y^{1}$ complements $\operatorname{ran} L^{2}$ in $Y^{2}$. Then, there exists an open neighborhood $X_{0}^{2} \subset U^{2}$ of $x_{0}$ such that $S^{2}\left(x^{2}\right)=0$ for $x^{2} \in X_{0}^{2}$ implies $x^{2} \in X^{1}$.

Proof. Let $N:=\operatorname{ker} L^{1}=\operatorname{ker} L^{2}$ be the finite-dimensional kernel. Take a closed linear subspace $V^{2}$ of $X^{2}$ which complements $N$ in $X^{2}$, and define the closed linear subspace $V^{1}:=V^{2} \cap X^{1}$ of $X^{1}$ so that $V^{1}$ complements $N$ in $X^{1}$. Also, let $R^{i}:=\operatorname{ran} L^{i} \subset Y^{i}$ be the closed finite-codimensional range. The linear subspace $W$ in the assumption is necessarily finite-dimensional and complements $\operatorname{ran} L^{1}$ in $Y^{1}$. We denote by $P_{R^{i}}: Y^{i} \rightarrow Y^{i}$ the bounded projection onto $R^{i}$ relative to $W$. Note that

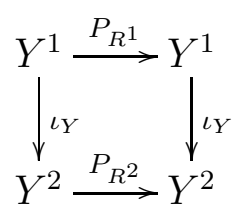

commutes. Consider the auxiliary operator $\varphi^{i}:=P_{R^{i}} \circ S^{i}: U^{i} \rightarrow R^{i}$. Since $\left.\frac{\partial \varphi^{i}}{\partial v^{i}}\right|_{0}: V^{i} \rightarrow R^{i}$ is an isomorphism of Banach spaces, the implicit function theorem implies that there exist open neighborhoods $0 \in N_{0} \subset N, 0 \in V_{0}^{i} \subset V^{i}$ and $C^{1}$ maps $\alpha^{i}: N_{0} \rightarrow V_{0}^{i}$ such that $N_{0} \times V_{0}^{i} \subset U^{i}$ and that

$$
\text { if } n \in N_{0}, v^{i} \in V_{0}^{i} \text {, then } \varphi^{i}\left(n+v^{i}\right)=0 \Longleftrightarrow v^{i}=\alpha^{i}(n) \text {. }
$$

We may assume $V_{0}^{1}=V_{0}^{2} \cap X^{1}$ since $V^{1}=V^{2} \cap X^{1}$.

We claim $\alpha^{1}=\alpha^{2}$. To show this, take an arbitrary $n \in N_{0}$. It follows from commutativity of diagrams (3.1), (3.2) that the equation $\varphi^{1}\left(n+\alpha^{1}(n)\right)=$ $P_{R^{1}} S^{1}\left(n+\alpha^{1}(n)\right)=0$ already implies $\varphi^{2}\left(n+\alpha^{1}(n)\right)=0$. Hence (3.3) with $i=2$ yields $\alpha^{1}(n)=\alpha^{2}(n)$.

The open neighborhood $X_{0}^{2}:=N_{0} \times V_{0}^{2} \subset U^{2}$ satisfies the desired property. Indeed, if $S^{2}\left(x^{2}\right)=0$ for $x^{2}=n+v^{2} \in X_{0}^{2}$, then $v^{2}=\alpha^{2}(n)=\alpha^{1}(n) \in V_{0}^{1} \subset X^{1}$, whence $x^{2} \in X^{1}$.

Theorem 3.2. Let $\varphi:\left(M^{m}, g\right) \rightarrow\left(N^{n}, h\right)$ be a harmonic Riemannian submersion of closed Riemannian manifolds. Assume $m \geq 3$ and the scalar curvature $\boldsymbol{s}_{g}$ of $g$ is constant along the fibers of $\varphi$. Assume also that $\Delta_{g} u+\frac{\boldsymbol{s}_{g}}{m-1} u=0$ for $u \in C^{k+2, \alpha}(M, g)$ implies $u$ is constant along the fibers of $\varphi$. Then, there exists an open neighborhood $U$ of $u \equiv 1$ in $C_{+}^{k+2, \alpha}(M, g)$ with the following property: If $u \in U$ and the scalar curvature of $u^{p_{m}-2} g$ is equal to $\boldsymbol{s}_{g}$, then $u$ is constant along the fibers of $\varphi$.

Proof. In the notation of Lemma 3.1, set $X^{2}=C^{k+2, \alpha}(M, g), Y^{2}=C^{k, \alpha}(M, g)$, $X^{1}=\left\{u \in C^{k+2, \alpha}(M, g) \mid u(p)=u(q)\right.$ if $\left.\varphi(p)=\varphi(q)\right\}, Y^{1}=X_{1} \cap Y^{2}, U^{i}=$ 
$\left\{u \in X^{i} \mid u>0\right\}, x_{0}=u \equiv 1, S^{i}(u)=-a_{m} \Delta_{g} u+s_{g}\left(u-u^{p_{m}-1}\right)$. It follows $L^{i}(u)=-a_{m}\left(\Delta_{g} u+\frac{\boldsymbol{s}_{g}}{m-1} u\right)$.

By hypothesis, $\operatorname{ker} L^{1}=\operatorname{ker} L^{2}=: W$. It follows from the Fredholm alternative (cf. Besse [3, p. 464]) and the essentially self-adjointness of $\Delta_{g}$ as the densely defined operator on $L^{2}(M, g)$ that $W$ is finite-dimensional and complements the closed linear subspace ran $L^{2}$ in $Y^{2}$.

We claim that $\operatorname{ran} L^{1}$ is a closed linear subspace which complements $W$ in $Y^{1}$. To see this, let $\boldsymbol{q}$ be the $C^{\infty}$ function on $N$ such that $\varphi^{*} \boldsymbol{q}=\boldsymbol{s}_{g}$, define $J: C^{k+2, \alpha}(N, h) \rightarrow C^{k, \alpha}(N, h)$ by $J(v)=-a_{m}\left(\Delta_{h} v+\frac{q}{m-1} v\right)$, and look at the direct sum decomposition $C^{k, \alpha}(N, h)=\operatorname{ker} J+\operatorname{ran} J$ into closed linear subspaces as for $(M, g)$ in the previous paragraph. Since $\varphi$ is a Riemannian submersion, the map $\varphi^{*}: C^{k, \alpha}(N, h) \rightarrow Y^{1}$ is an isomorphism of Banach spaces. Furthermore, since $\varphi$ is Laplacian-commuting, $\varphi^{*}(\operatorname{ker} J)=W, \varphi^{*}(\operatorname{ran} J)=\operatorname{ran} L^{1}$. Hence $Y^{1}=$ $W+\operatorname{ran} L^{1}$ is a direct sum decomposition into closed linear subspaces.

Apply Lemma 3.1 to get an open neighborhood $X_{0}^{2}=: U$ of $x_{0}=u \equiv 1$ in $U^{2}=C_{+}^{k+2, \alpha}(M, g)$ such that $S^{2}(u)=-a_{m} \Delta_{g} u+s_{g}\left(u-u^{p_{m}-1}\right)=0$ for $u \in U$ implies $u$ is constant along the fibers of $\varphi$. This $U$ has the desired property since the scalar curvature of $u^{p_{m}-2} g$ is equal to $u^{1-p_{m}}\left(-a_{m} \Delta_{g} u+\boldsymbol{s}_{g} u\right)$.

\section{Existence of bifurcation points}

A nonzero crossing number detects bifurcation for a potential operator, while a nonzero even topological degree for an operator without potential does not always imply bifurcation (cf. Nirenberg [14, p. 46]). There are various such bifurcation theorems for potential operators in the literature (cf. Krasnosel'skii [12], Rabinowitz [17], Kielhöfer [9], [10, p. 193, 240]). For a potential operator whose linearization is diagonalizable, the Lyapunov-Schmidt reduction due to SmollerWasserman [20] is convenient.

Theorem 4.1. Let $I$ be an open interval of $\mathbb{R}$ and $\varphi:\left(M^{m}, g(t)\right) \rightarrow\left(N^{n}, h\right)$ a harmonic Riemannian submersion of closed Riemannian manifolds for all $t \in I$, where $\varphi, M, N, h$ are fixed. Suppose $m \geq 3, g(t)$ depends on $t C^{\infty}$ smoothly, and each $g(t)$ has constant scalar curvature. If $\boldsymbol{s}_{g\left(t_{\star}\right)} /(m-1)$ is a nonzero eigenvalue of $-\Delta_{h}$ for $t_{\star} \in I$, and if there are sequences $\left\{r_{j}\right\}_{j \geq 1},\left\{s_{j}\right\}_{j \geq 1} \subset I$ such that

$$
\begin{gathered}
r_{j}<t_{\star}<s_{j}, \quad \lim _{j \rightarrow \infty} r_{j}=\lim _{j \rightarrow \infty} s_{j}=t_{\star}, \\
\left(\boldsymbol{s}_{g\left(r_{j}\right)}-\boldsymbol{s}_{g\left(t_{\star}\right)}\right)\left(\boldsymbol{s}_{g\left(s_{j}\right)}-\boldsymbol{s}_{g\left(t_{\star}\right)}\right)<0,
\end{gathered}
$$

then $g\left(t_{\star}\right)$ is a bifurcation point for the constant scalar curvature equation along $\{g(t)\}_{t \in I}$. 
Remark 4.2. If $\boldsymbol{s}_{g(t)}$ is monotone near $t_{\star}$, then there exist such sequences $\left\{r_{j}\right\}_{j \geq 1}$, $\left\{s_{j}\right\}_{j \geq 1} \subset I$ as in the assumption of Theorem 4.1.

Proof. Define the nonlinear operator $T: C_{+}^{k+2, \alpha}(N, h) \times I \rightarrow C^{k, \alpha}(N, h)$ by

$$
T(v, t)=-a_{m} \Delta_{h} v+\boldsymbol{s}_{g(t)}\left(v-v^{p_{m}-1}\right),
$$

which has the potential $F: C_{+}^{k+2, \alpha}(N, h) \times I \rightarrow \mathbb{R}$,

$$
F(v, t)=\int_{N} \frac{a_{m}}{2}|d v|^{2}+\boldsymbol{s}_{g(t)}\left(\frac{v^{2}}{2}-\frac{v^{p_{m}}}{p_{m}}\right) d \mu_{h}
$$

with respect to the inner product of $L^{2}(N, h)$. Note that

$$
\left.\frac{\partial T}{\partial v}\right|_{(v \equiv 1, t)} f=-a_{m}\left(\Delta_{h} f+\frac{\boldsymbol{s}_{g(t)}}{m-1} f\right)
$$

holds for all $f \in C^{k+2, \alpha}(N, h)$. Hence, with respect to the functional $F(\cdot, t)$ for each fixed $t \in I, v \equiv 1$ is a degenerate critical point if $\boldsymbol{s}_{g(t)} /(m-1)$ is an eigenvalue of $-\Delta_{h}$, and if $v \equiv 1$ is nondegenerate, then its Morse index is equal to the number of eigenvalues for $-\Delta_{h}$ strictly less than $\boldsymbol{s}_{g(t)} /(m-1)$, counted with multiplicity.

By hypothesis, $v \equiv 1$ is a degenerate critical point for $F\left(\cdot, t_{\star}\right)$. On the other hand, since the spectrum of $-\Delta_{h}$ is discrete and since $\boldsymbol{s}_{g(t)}$ depends on $t$ continuously, (4.1) and (4.2) imply that $v \equiv 1$ is nondegenerate with respect to $F\left(\cdot, r_{j}\right)$ and $F\left(\cdot, s_{j}\right)$ for all $r_{j}, s_{j}$ with $j$ sufficiently large. For such $j,(4.2)$ implies that the Morse indices of $v \equiv 1$ with respect to $F\left(\cdot, r_{j}\right), F\left(\cdot, s_{j}\right)$ are different. Therefore, applying the bifurcation theorem of Smoller-Wasserman [20, Theorem 2.1] to the gradient operator $T$, we see that for every $j$ large there exists a real number $t_{j} \in\left(r_{j}, s_{j}\right)$ such that $(v \equiv 1, t)$ is a bifurcation point for the equation $T=0$. It then follows from (4.1) that $t_{\star}=\lim _{j \rightarrow \infty} r_{j}=\lim _{j \rightarrow \infty} s_{j}$. Hence $\left(v \equiv 1, t_{\star}\right)$ is a bifurcation point because the set $\{t \in I \mid(v \equiv 1, t)$ is a bifurcation point for $T=0\}$ is closed in $I$. That is, there exist sequences $\left\{t_{j}\right\}_{j \geq 1} \subset I,\left\{v_{j}\right\}_{j \geq 1} \subset C_{+}^{k+2, \alpha}(N, h)$ such that $v_{j} \not \equiv 1, T\left(v_{j}, t_{j}\right)=0$ for all $j$ and that $t_{j} \rightarrow t_{\star}, v_{j} \rightarrow 1$ as $j \rightarrow \infty$.

Since $\varphi$ is Laplacian-commuting (cf. Sect. 5), if $T(v, t)=0$ for some $v \in$ $C_{+}^{k+2, \alpha}(N, h)$ and $t \in I$, then

$$
-a_{m} \Delta_{g(t)} u+\boldsymbol{s}_{g(t)}\left(u-u^{p_{m}-1}\right)=0
$$

for $u=\varphi^{*} v \in C_{+}^{k+2, \alpha}(M, g(t))$. In particular, $u_{j}:=\varphi^{*} v_{j} \not \equiv 1$ satisfies (4.3) for all $j \geq 1$. Also, $u_{j} \rightarrow 1$ in $C^{k+2, \alpha}\left(M, g\left(t_{\star}\right)\right)$ since the linear map $\varphi^{*}: C^{k+2, \alpha}(N, h) \rightarrow$ $C^{k+2, \alpha}\left(M, g\left(t_{\star}\right)\right)$ is continuous. Finally, $u_{j} \not \equiv 1$ implies that $u_{j}$ is nonconstant since $\boldsymbol{s}_{g\left(t_{\star}\right)}$ is nonzero by hypothesis. Hence $g\left(t_{\star}\right)$ is a bifurcation point along $\{g(t)\}_{t \in I}$ according to Definition 2.1. 


\section{The canonical variation of a Riemannian submersion with totally geodesic fibers}

Recall that, for a smooth map $\varphi:(M, g) \rightarrow(N, h)$ of Riemannian manifolds, the following are equivalent:

(1) $\varphi$ is a Riemannian submersion each of whose fiber is a minimal submanifold of $(M, g)$.

(2) $\varphi$ is a Riemannian submersion and a harmonic map at the same time.

(3) $\varphi$ is Laplacian-commuting. That is, $\varphi^{*} \circ \Delta_{h}=\Delta_{g} \circ \varphi^{*}$.

See Eells-Sampson [6], Watson [22]. Such a map $\varphi$ is called a harmonic Riemannian submersion. In particular, a Riemannian submersion with totally geodesic fibers is harmonic.

Consider the canonical variation

$$
\left(F^{k}, t \hat{g}\right) \rightarrow\left(M^{m}, g(t)\right) \stackrel{\varphi}{\rightarrow}\left(N^{n}, h\right)
$$

of a Riemannian submersion with totally geodesic fibers, $m \geq 3$. Assume henceforth that $g(t)$ has constant scalar curvature for every $t>0$; this is equivalent to saying that both the typical fiber $(F, \hat{g})$ and the base space $(N, h)$ have constant scalar curvature (cf. [15, Proposition 3.2]). Let

$$
\begin{aligned}
B & =\left\{t>0 \mid g(t) \text { is a bifurcation point for the csc equation along }\{g(t)\}_{t>0}\right\}, \\
D & =\left\{t>0 \mid \lambda=\boldsymbol{s}_{g(t)} /(m-1) \text { for a nonzero } \lambda \in \operatorname{Spec}\left(-\Delta_{g(t)}\right)\right\}, \\
D_{\text {hor }} & =\left\{t>0 \mid \lambda=s_{g(t)} /(m-1) \text { for a nonzero } \lambda \in \operatorname{Spec}\left(-\Delta_{h}\right)\right\},
\end{aligned}
$$

where $B \subset D, D_{\text {hor }} \subset D$. On the one hand, every eigenvalue $\lambda \geq 0$ of $-\Delta_{g(t)}$ can be written as

$$
\lambda=b+\hat{\lambda} / t
$$

where $b$ and $\hat{\lambda}$ are respectively some eigenvalues of $-\Delta_{\text {hor }}$ and $-\Delta_{\hat{g}}$. Here, $\Delta_{\text {hor }}$ denotes the horizontal Laplacian (cf. [2], [4, Remark 3.3]). On the other hand,

$$
\boldsymbol{s}_{g(t)}=\boldsymbol{s}_{h}+\boldsymbol{s}_{\hat{g}} / t-t|A|^{2},
$$

where $A$ is the O'Neill's integrability tensor (see [3, (9.70d)]). Hence, for $t>0$, $t \in D$ if and only if there exist some $b \in \operatorname{Spec}\left(-\Delta_{\text {hor }}\right), \hat{\lambda} \in \operatorname{Spec}\left(-\Delta_{\hat{g}}\right)$ such that $b+\hat{\lambda} \in \operatorname{Spec}\left(-\Delta_{g(1)}\right) \backslash\{0\}$ and

$$
\left(b-\frac{s_{h}}{m-1}\right)+\frac{1}{t}\left(\hat{\lambda}-\frac{s_{\hat{g}}}{m-1}\right)+t \frac{|A|^{2}}{m-1}=0 .
$$


Proposition 5.1. The set $D$ of degeneracy instants is not discrete if and only if

$$
\begin{aligned}
& |A|=0, \\
& b-\frac{\boldsymbol{s}_{h}}{m-1}=0 \text { for some } b \in \operatorname{Spec}\left(-\Delta_{\text {hor }}\right), \\
& \hat{\lambda}-\frac{\boldsymbol{s}_{\hat{g}}}{m-1}=0 \text { for some } \hat{\lambda} \in \operatorname{Spec}\left(-\Delta_{\hat{g}}\right), \\
& b+\hat{\lambda} \in \operatorname{Spec}\left(-\Delta_{g(1)}\right) \backslash\{0\}
\end{aligned}
$$

holds, in which case $D$ is necessarily equal to $(0, \infty)$.

Remark 5.2. The condition $|A|=0$ amounts to saying that $(M, g(1))$ is locally the direct product $(N, h) \times(F, \hat{g})$. If $(M, g(1))$ is globally the direct product $(N, h) \times(F, \hat{g})$, then $(5.3)$ holds if and only if $(N, h),(F, \hat{g})$ is a non-degenerate pair in the sense of de Lima-Piccione-Zedda [7, p. 269].

Proof. If (5.3) holds, then it follows from (5.2) that $D=(0, \infty)$, which is not discrete.

Conversely, assume that (5.3) does not hold. We prove discreteness of $D$ by showing that every convergent sequence in $D$ has a constant subsequence. Let $\left\{t_{l}\right\}_{l \geq 1} \subset D$ be convergent. By definition of $D$, there is a nonzero eigenvalue $\lambda(l)$ of $-\Delta_{g\left(t_{l}\right)}$ such that $\lambda(l)=s_{g\left(t_{l}\right)} /(m-1)$ for each $l \geq 1$. We write $\lambda(l)=b(l)+\hat{\lambda}(l) / t_{l}$ for some $b(l) \in \operatorname{Spec}\left(-\Delta_{\text {hor }}\right), \hat{\lambda}(l) \in \operatorname{Spec}\left(-\Delta_{\hat{g}}\right)$.

We claim that, after taking a subsequence, both $\hat{\lambda}(l)$ and $b(l)$ are constant in $l$. Note that the corresponding sequence $\boldsymbol{s}_{g\left(t_{l}\right)}$ converges by smoothness in $t$ of the family $\{g(t)\}_{t>0}$. Hence $\lambda(l)=\boldsymbol{s}_{g\left(t_{l}\right)} /(m-1)$ converges. Since $0 \leq$ $\hat{\lambda}(l)=t_{l}(\lambda(l)-b(l)) \leq t_{l} \lambda(l)$ by nonnegativity of $\operatorname{Spec}\left(-\Delta_{\hat{g}}\right)$ and $\operatorname{Spec}\left(-\Delta_{\text {hor }}\right)$, it follows from discreteness of $\operatorname{Spec}\left(-\Delta_{\hat{g}}\right)$ that the bounded sequence $\hat{\lambda}(l)$ has a constant subsequence. In particular, $b(l)=\lambda(l)-\hat{\lambda}(l) / t_{l}$ also converges as $l \rightarrow \infty$. The sequence $b(l)+\hat{\lambda}(l)$ of eigenvalues for $-\Delta_{g(1)}$ is then convergent and therefore eventually constant by discreteness of $\operatorname{Spec}\left(-\Delta_{g(1)}\right)$. Therefore, $b(l)$ is also constant for $l$ large. Note that the spectrum of the horizontal Laplacian may not be discrete [2, Warning 3.2].

Assume henceforth that $\hat{\lambda}(l), b(l)$ are constant in $l$. Recall that $\lambda(l)=s_{g\left(t_{l}\right)} /(m-$ 1 ) is equivalent to

$$
\left(b(l)-\frac{\boldsymbol{s}_{h}}{m-1}\right)+\frac{1}{t_{l}}\left(\hat{\lambda}(l)-\frac{\boldsymbol{s}_{\hat{g}}}{m-1}\right)+t_{l} \frac{|A|^{2}}{m-1}=0 .
$$

Since (5.3) does not hold, the polynomial equation

$$
\left(b(l)-\frac{\boldsymbol{s}_{h}}{m-1}\right)+\frac{1}{t}\left(\hat{\lambda}(l)-\frac{\boldsymbol{s}_{\hat{g}}}{m-1}\right)+t \frac{|A|^{2}}{m-1}=0
$$


of $t$ with constant coefficients has at most two roots. It follows that $t_{l}$ must be one of these two roots, and we conclude that $\left\{t_{l}\right\}_{l \geq 1}$ has a subsequence which is eventually constant, showing discreteness of $D$.

Lemma 5.3. Suppose the first nonzero eigenvalue of $-\Delta_{\hat{g}}$ satisfies

$$
\hat{\lambda}_{1}>\boldsymbol{s}_{\hat{g}} /(m-1) \text {. }
$$

Then, for every $t>0$ sufficiently small, $\Delta_{g(t)} f+\frac{s_{g(t)}}{m-1} f=0$ for a nonzero $f \in$ $C^{k+2, \alpha}(M, g(t))$ implies $f$ is constant along the fibers of $\varphi$ and $\frac{\boldsymbol{s}_{g(t)}}{m-1} \in \operatorname{Spec}\left(-\Delta_{h}\right)$.

Remark 5.4. Under the assumption (5.4), the set $D$ is discrete by Proposition 5.1. Also, (5.4) is fulfilled if $\hat{\lambda}_{1} \geq \boldsymbol{s}_{\hat{g}} /(k-1)>0$ and $n \geq 1$ or if $\boldsymbol{s}_{\hat{g}} \leq 0$. When $k \geq 3$, the inequality $\hat{\lambda}_{1} \geq \boldsymbol{s}_{\hat{g}} /(k-1)$ is equivalent to the stability of the critical point $\hat{g}$ with respect to the Einstein-Hilbert functional restricted to its conformal class.

Proof. For a fixed $t>0$, suppose $\Delta_{g(t)} f+\frac{s_{g(t)}}{m-1} f=0$ holds for a nonzero $f \in$ $C^{k+2, \alpha}(M, g(t))$. Then $\frac{\boldsymbol{s}_{g(t)}}{m-1}=b+\hat{\lambda} / t$ for some $b \in \operatorname{Spec}\left(-\Delta_{\text {hor }}\right), \hat{\lambda} \in \operatorname{Spec}\left(-\Delta_{\hat{g}}\right)$. Observe from (5.1) that, if $\hat{\lambda}>0$, then

$$
0=\left(b-\frac{\boldsymbol{s}_{h}}{m-1}\right)+\frac{1}{t}\left(\hat{\lambda}-\frac{\boldsymbol{s}_{\hat{g}}}{m-1}\right)+t \frac{|A|^{2}}{m-1} \geq-\frac{\boldsymbol{s}_{h}}{m-1}+\frac{1}{t}\left(\hat{\lambda}_{1}-\frac{\boldsymbol{s}_{\hat{g}}}{m-1}\right) .
$$

That is, if

$$
\frac{\boldsymbol{s}_{h}}{m-1}<\frac{1}{t}\left(\hat{\lambda}_{1}-\frac{\boldsymbol{s}_{\hat{g}}}{m-1}\right)
$$

then $\hat{\lambda}=0$.

By hypothesis, (5.5) holds for every $t>0$ sufficiently small. Therefore, for such a $t>0, \Delta_{g(t)} f+\frac{\boldsymbol{s}_{g(t)}}{m-1} f=0$ for a nonzero $f \in C^{k+2, \alpha}(M, g(t))$ implies that $f$ is an eigenfunction of $-\Delta_{\text {hor }}$ and is constant along the fibers of $\varphi$. Since $\varphi$ is Laplacian-commuting, $\frac{\boldsymbol{s}_{g(t)}}{m-1} \in \operatorname{Spec}\left(-\Delta_{h}\right)$.

Theorem 5.5. If $\boldsymbol{s}_{\hat{g}}>0$, then there exists a sequence $\left\{b_{l}\right\}_{l \geq 1} \subset(0, \infty)$ such that $b_{l+1}<b_{l}$ for all $j, \lim _{l \rightarrow \infty} b_{l}=0$, and

$$
D_{\text {hor }}=\left\{b_{l} \mid l \geq 1\right\} \subset B .
$$

If moreover $\lambda_{1}\left(-\Delta_{\hat{g}}\right)>\boldsymbol{s}_{\hat{g}} /(m-1)$, then there exists such a real number $\varepsilon>0$ that

$$
D_{\text {hor }} \cap(0, \varepsilon)=D \cap(0, \varepsilon)=B \cap(0, \varepsilon)
$$

and that the following holds: If $\left\{t_{j}\right\}_{j \geq 1}$ is a sequence of $(0, \infty)$ such that $\lim _{j \rightarrow \infty} t_{j}=$ $t_{\star} \in B \cap(0, \varepsilon)$ and $\left\{u_{j}\right\}_{j \geq 1}$ is a sequence of $C_{+}^{k+2, \alpha}\left(M, g\left(t_{\star}\right)\right)$ such that $\tilde{g}_{j}=$ $u_{j}^{p_{m}-2} g\left(t_{j}\right)$ has constant scalar curvature for all $j \geq 1$ and $\lim _{j \rightarrow \infty} u_{j}=1$, then $u_{j}$ is constant along the fibers of $\varphi$ for $j$ sufficiently large. 
Proof. Assume $\boldsymbol{s}_{\hat{g}}>0$. Then $\boldsymbol{s}_{g(t)}=\boldsymbol{s}_{h}+\boldsymbol{s}_{\hat{g}} / t-t|A|^{2}$ is strictly monotone in $t$ and $\lim _{t \rightarrow 0} \boldsymbol{s}_{g(t)}=\infty$. Hence there exists a sequence $\left\{b_{j}\right\}_{j \geq 1} \subset(0, \infty)$ such that $b_{j+1}<b_{j}, b_{j} \rightarrow 0$, and $D_{\text {hor }}=\left\{b_{j} \mid j \geq 1\right\}$. Explicitly, $b_{j}$ is the solution of the equation $\lambda_{i+j}\left(-\Delta_{h}\right)=s_{g(t)} /(m-1)$ for some $i \geq 0$, where $\lambda_{i+j}\left(-\Delta_{h}\right)$ is the $(i+j)$-th eigenvalue counted without multiplicity. We apply Theorem 4.1 for each $b_{j}=: t_{\star}$ to see $D_{\text {hor }} \subset B$. This shows (5.6). Note that we allow the larger set $D$ of degeneracy instants to be the whole $(0, \infty)$. See Proposition 5.1.

Assume moreover $\lambda_{1}\left(-\Delta_{\hat{g}}\right)>\boldsymbol{s}_{\hat{g}} /(m-1)$, so that in particular $D$ is discrete. For a small $\varepsilon>0$, we see $D \cap(0, \varepsilon) \subset D_{\text {hor }} \cap(0, \varepsilon)$ from Lemma 5.3. This together with (5.6) implies (5.7). The rest of the statement follows from Theorem 3.2 applied to $g\left(t_{\star}\right)$ for each fixed $t_{\star} \in B \cap(0, \varepsilon)$.

Corollary 5.6. Assume $\lambda_{1}\left(-\Delta_{\hat{g}}\right)>\boldsymbol{s}_{\hat{g}} /(m-1)>0$. If either $\boldsymbol{s}_{h} \leq 0,|A|>0$, or $(M, g(1))=(N, h) \times(F, \hat{g})$ and $\lambda_{1}\left(-\Delta_{h}\right)>\boldsymbol{s}_{h} /(m-1)>0$, then there exists a compact interval $I$ of $(0, \infty)$ such that $D \cap((0, \infty) \backslash I)=B \cap((0, \infty) \backslash I)$.

Proof. If $\boldsymbol{s}_{h} \leq 0$, then we can replace $(0, \varepsilon)$ in $(5.7)$ with $(0, \infty)$ because $(5.5)$ holds for all $t>0$. If $|A|>0$, then $\lim _{t \rightarrow \infty} \boldsymbol{s}_{g(t)}=-\infty$ and no degeneration occurs for $t$ sufficiently large. If $(M, g(1))=(N, h) \times(F, \hat{g})$ and $\lambda_{1}\left(-\Delta_{h}\right)>\boldsymbol{s}_{h} /(m-1)>0$, then we interchange the role of $(N, h),(F, \hat{g})$ and apply Theorem 5.5.

An open problem in view of Proposition 5.1 is to ask whether $D=B$ holds whenever $D$ is discrete. Corollary 5.6 provides a partial answer outside the compact interval $I$. In the case of product manifolds, de Lima-Piccione-Zedda [7] addresses this question. Bettiol-Piccione [4] essentially shows $D_{\text {hor }}=D=B$ in the case of Hopf fibrations. Last but not least, we remark that Theorem 3.2 implies that, in the case of quaternionic Hopf fibrations, no symmetry-breaking bifurcation occurs except at the round metric.

\section{Acknowledgements}

N. Otoba is supported by the DFG (Deutsche Forschungsgemeinschaft), SFB 1085 Higher Invariants. J. Petean is supported by Grant 220074 Fondo Sectorial de Investigación para la Educación CONACYT.

\section{References}

[1] Aubin, T. "Équations différentielles non linéaires et probléme de Yamabe concernant la courbure scalaire." J. Math. Pures Appl. 55, no. 3 (1976): 269-296.

[2] Bérard-Bergery, L., and J-P. Bourguignon. "Laplacians and Riemannian submersions with totally geodesic fibres." Illinois J. Math. 26, no. 2 (1982): 181200. 
[3] Besse, A. Einstein manifolds, Reprint of the 1987 edition. Berlin: SpringerVerlag, 2008.

[4] Bettiol, R., and P. Piccione. "Bifurcation and local rigidity of homogeneous solutions to the Yamabe problem on spheres." Calc. Var. Partial Differential Equations 47, no. 3-4 (2013): 789-807.

[5] Bettiol, R., and P. Piccione. "Multiplicity of solutions to the Yamabe problem on collapsing Riemannian submersions." Pacific J. Math. 266, no. 1 (2013): $1-21$.

[6] Eells, J., and J. H. Sampson. "Harmonic Mappings of Riemannian Manifolds." Amer. J. Math. 86, no. 1 (1964): 109-160.

[7] de Lima, L. L., P. Piccione, and M. Zedda. "On bifurcation of solutions of the Yamabe problem in product manifolds." Ann. Inst. H. Poincaré Anal. Non Linéaire 29 (2012): 261-277.

[8] Henry, G., and J. Petean. "Isoparametric hypersurfaces and metrics of constant scalar curvature." Asian J. Math. 18 (2014): 53-68.

[9] Kielhöfer, H. "A bifurcation theorem for potential operators." J. Funct. Anal. 77, no. 1 (1988): 1-8.

[10] Kielhöfer, H. Bifurcation theory, second ed. New York: Springer, 2012.

[11] Kobayashi, O. "On large scalar curvature." Research Report, Dept. Math., Keio Univ. 85/011, 1985.

[12] Krasnosel'skii, M. A. Topological methods in the theory of nonlinear integral equations. New York: The Macmillan Co., 1964.

[13] Jin, Q., Y.Y. Li, and H. Xu. "Symmetry and asymmetry: the method of moving spheres." Adv. Differential Equations 13 (2008): 601-640.

[14] Nirenberg, L. Topics in nonlinear functional analysis, Revised reprint of the 1974 original. New York: Courant Institute of Mathematical Sciences, 1974.

[15] Otoba, N., and J. Petean. "Metrics of constant scalar curvature on sphere bundles." Differential Geom. Appl. 46 (2016): 146-163.

[16] Petean, J. "Metrics of constant scalar curvature conformal to Riemannian products." Proc. Amer. Math. Soc. 138, no. 8 (2010): 2897-2905.

[17] Rabinowitz, P. H. "A bifurcation theorem for potential operators." J. Funct. Anal. 25, no. 4 (1977): 412-424. 
[18] Schoen, R. "Conformal deformation of a Riemannian metric to constant scalar curvature." J. Differential. Geometry 20, no. 2 (1984): 479-495.

[19] Schoen, R. "Variational theory for the total scalar curvature functional for Riemannian metrics and related topics." Lecture Notes in Math. vol. 1365, Springer (1989): 120-154.

[20] Smoller, J., and A.G. Wasserman. "Bifurcation and symmetry breaking." Invent. Math. 100 (1990): 63-95.

[21] Trudinger, N. "Remarks concerning the conformal deformation of Riemannian structures on compact manifolds." Annali Scuola Norm. Sup. Pisa 22 (1968): $265-274$.

[22] Watson, B. "Manifold maps commuting with the Lapacian." J. Differential Geometry 8 (1973): 85-94.

[23] Yamabe, H. "On a deformation of Riemannian structures on compact manifolds." Osaka J. Math. 12 (1960): 21-37. 